\title{
Association of Insulin Resistance and Hyperinsulinemia with Disturbed Lipid Metabolism in Patients with Essential Hypertension
}

\author{
Jun Agata, Yoshinori Miyazaki, Mikio Takada, Hideyuki Murakami, Atsushi Masuda, \\ Tetuji Miura, Nobuyuki Ura, and Kazuaki Shimamoto
}

\begin{abstract}
To clarify the association of insulin resistance and hyperinsulinemia with lipid metabolism in patients with essential hypertension (EHT), we used the euglycemic hyperinsulinemic glucose clamp technique (GC) and the 75-g oral glucose tolerance test (OGTT) to compare the characteristics of glucose and lipid metabolism in insulin-resistant patients with essential hypertension (EHT-R) with those in insulin-nonresistant patients with essential hypertension (EHT-N) and normotensive subjects (NT). Twenty-eight NT and 42 EHT whose body mass index (BMI) was less than $28 \mathrm{~kg} / \mathrm{m}^{2}$ were studied to eliminate the effects of obesity on insulin sensitivity and lipid metabolism. Insulin sensitivity was evaluated by GC and expressed as metabolic clearance rate of glucose $\left(M\right.$ value, $\left.\mathrm{mg} / \mathrm{m}^{2} / \mathrm{min}\right)$. Mean -1 SD of the $M$ value in NT $\left(145.0 \mathrm{mg} / \mathrm{m}^{2} / \mathrm{min}\right)$ was chosen as the cutoff point for insulin resistance. On the basis of this value, $33.3 \%$ of the EHT were EHT-R. There was no significant difference in age or BMI among the three groups. Blood samples were collected before GC to measure levels of total cholesterol (TC), triglyceride (TG), free fatty acid (FFA), and HDL cholesterol (HDL-C).

EHT-R had significantly higher levels of fasting blood sugar, fasting immunoreactive insulin, insulin at $120 \mathrm{~min}$ (IRI-120), and summation of insulin or blood sugar (BS) during the OGTT, as compared with NT and EHT-N. EHT-R also had significantly higher FFA and TG than the other two groups, while there was no difference in FFA or TG between EHT-N and NT. TC and HDL-C were similar in the three groups. There was either a significant negative correlation, or a trend toward negative correlation, between $M$ value and FFA $(r=-0.50, p<0.05)$ or TG $(r=-0.24, p<0.1)$. There were significant positive correlations between IRI-120 and FFA $(r=0.35, p<0.05)$ or TG $(r=0.29, p<0.05)$. There was a positive correlation $(r=-0.36, p<0.01)$ between $\Sigma$ BS and FFA, while no other significant relation was found between $\Sigma$ BS and serum lipids.

In summary, (i) $33.3 \%$ of EHT were found to be insulin resistant, when insulin resistance was defined as $M$ value $<145.0 \mathrm{mg} / \mathrm{m}^{2} / \mathrm{min}$, i.e., mean - 1SD of the $M$ value of NT; (ii) these EHT-R had higher levels of BS, insulin, FFA, and TG than did NT and EHT-N; (iii) EHT-N showed no difference in the levels of BS, insulin, or lipid, as compared with NT; and (iv) the levels of FFA and of TG correlated negatively with insulin sensitivity and positively with the insulin level during the OGTT.

These results suggest that disturbances of glucose and lipid metabolism in EHT may be related to both insulin resistance and compensatory hyperinsulinemia, and that EHT-R may have more risk factors for arteriosclerotic complications than EHT-N. (Hypertens Res 1998; 21: 57-62)
\end{abstract}

Key Words: insulin resistance, hyperinsulinemia, lipid disturbance, essential hypertension

Insulin resistance and hyperinsulinemia are often associated with obesity, glucose intolerance, and dyslipidemia. Recently, insulin resistance has also been linked to essential hypertension (1-6). Hyperinsulinemia, a reaction that attempts to compensate for insulin resistance, is thought to cause and maintain high blood pressure by promoting sodium reabsorption in the renal tubules $(7-10)$ and by stimulating the sympathetic nervous system and reninangiotensin system $(9,11-14)$.

Patients with essential hypertension (EHT) show a wide range of insulin sensitivity. Lind et al. (15) reported that $27 \%$ of hypertensive subjects are insulin resistant and display a high degree of clustering of glucose and lipid metabolic disturbances. However, their study was unable to determine whether or not insulin resistance itself was directly responsible for these metabolic disturbances in EHT, because their insulin-resistant patients with essential hypertension (EHT-R) had a higher body mass index (BMI) than the insulin-non-resistant patients with essential hypertension (EHT-N). It has been reported that obesity per se can be associated with lipid disturbances or insulin resistance.

From the Second Department of Internal Medicine, Sapporo Medical University School of Medicine.

Address for Reprints: Jun Agata, M.D., The Second Department of Internal Medicine, Sapporo Medical University School of Medicine, S-1, W-16, Chuo-ku, Sapporo 060-0061, Japan.

Received December 8, 1997; accepted in revised form February 3, 1998. 
To clarify the association of insulin resistance and hyperinsulinemia with glucose and lipid metabolism in EHT, we examined metabolic variables in EHT$\mathrm{R}$, as compared with BMI-matched EHT-N and normotensive subjects (NT).

\section{Material and Methods}

\section{Subjects}

Twenty-eight NT and 42 EHT with a BMI less than $28 \mathrm{~kg} / \mathrm{m}^{2}$ were studied. Blood pressure was measured with a mercury sphygmomanometer, with the patient in a supine position after $10 \mathrm{~min}$ of rest. The subjects were admitted to the hospital and given a regular diet $(2,000 \mathrm{kcal} / \mathrm{d})$ containing $310 \mathrm{~g}$ of carbohydrate, $50 \mathrm{~g}$ of fat, $80 \mathrm{~g}$ of protein, 120 mmol of sodium, and $75 \mathrm{mmol}$ of potassium, for more than $1 \mathrm{wk}$. All drugs that may affect insulin sensitivity were discontinued at least $2 \mathrm{wk}$ before the study in subjects receiving medical treatment. All subjects were screened by routine physiological and laboratory examinations for complications such as endocrine or metabolic disturbances, cerebrovascular or cardiovascular disease, and renal disease. No subject had evidence of complications. Insulin sensitivity was evaluated as the $M$ value (metabolic clearance rate of glucose, $\mathrm{mg} / \mathrm{m}^{2} / \mathrm{min}$ ) with the euglycemic hyperinsulinemic glucose clamp technique. Mean -1SD of the $M$ value in NT was chosen as the cutoff point for insulin resistance. At this point, EHT were divided into two groups: EHT-R and EHT-N. This study was approved by the ethical committee of Sapporo Medical University, and informed consent was obtained from each subject.

\section{Insulin Sensitivity Study}

The 2-h euglycemic hyperinsulinemic glucose clamp technique (16) was carried out from 9:00 AM after an overnight fast. Briefly, arterialized blood was continuously withdrawn at a rate of $2 \mathrm{ml} / \mathrm{h}$ through a double-lumen catheter for glucose analysis. A contralateral antecubital vein was cannulated with a No. 20 plastic cannula for infusion of insulin and glucose. An artificial endocrine pancreas (model STG-22, Nikkiso, Tokyo, Japan) was used for continuous infusion of insulin, done while monitoring the blood glucose level and the amount of glucose infused to clamp glucose levels at the basal state. Insulin (Actrapid Human, Novo Industries, Copenhagen, Denmark) was infused at a rate of $40 \mathrm{mU} /$ $\mathrm{min} / \mathrm{m}^{2}$ of body surface area. Throughout the study, euglycemia was maintained by an infusion of $20 \%$ glucose solution, the infusion rate of which was adjusted according to the blood glucose level. Insulin sensitivity was assessed as the mean infusion rate of glucose ( $M$ value) for the last $30 \mathrm{~min}$ during which the infusion rate had been stable. To reduce the influence of obesity on insulin sensitivity, the $M$ value was expressed as $\mathrm{mg}$ of glucose $/ \mathrm{m}^{2}$ of body surface area.

Serum Lipid Profiles

At 9:00 AM just before the glucose clamp study, we collected blood samples and measured serum lipid profiles, including total cholesterol (TC), triglyceride (TG), free fatty acid (FFA), and HDL cholesterol (HDL-C).

\section{Oral Glucose Tolerance Test}

After an overnight fast, all subjects underwent a 75$\mathrm{g}$ oral glucose tolerance test. Blood samples were collected before the test and 30,60, 90, and 120 min after oral glucose loading to measure blood sugar (BS) and plasma immunoreactive insulin levels (IRI).

\section{Laboratory Investigations}

BS levels were measured by the glucose oxidase method. IRI was measured by radioimmunoassay (Insulin RIA bead, Dainabot, Tokyo, Japan). TC, TG, FFA, and HDL-C were estimated with enzymatic methods.

\section{Statistical Analysis}

All data are expressed as means \pm SEM. Paired and unpaired Student's $t$-tests and Chi-square test were used to assess the statistical significance of differences between the groups. Linear regression analysis was used to determine the correlation between the groups. All calculated probability values less than 0.05 were considered to indicate statistical significance.

\section{Results}

In this study, there was no significant difference in age or BMI between NT $(47.0 \pm 2.3$ yr old, $23.7 \pm$ $\left.0.4 \mathrm{~kg} / \mathrm{m}^{2}\right)$ and EHT $(51.7 \pm 2.1 \mathrm{yr}$ old, $24.1 \pm 0.4$ $\mathrm{kg} / \mathrm{m}^{2}$ ). EHT had a significantly lower $M$ value than NT $\left(160.7 \pm 7.4\right.$ vs. $\quad 181.7 \pm 7.3 \mathrm{mg} / \mathrm{m}^{2} / \mathrm{min}, \quad p<$ 0.05 , EHT vs. NT), as well as a wide range of $M$ values (minimum, $64.4 \mathrm{mg} / \mathrm{m}^{2} / \mathrm{min}$; maximum, 257.1 $\mathrm{mg} / \mathrm{m}^{2} / \mathrm{min}$ ). When EHT were divided into two groups at a cutoff $M$ value of $145.0 \mathrm{mg} / \mathrm{m}^{2} / \mathrm{min}$ (mean -1SD of $M$ value in NT), $33.3 \%$ of EHT were classified as EHT-R. As shown in Table 1, there was no significant differences in age or BMI among NT, EHT-N, and EHT-R. There was a significant difference in sex distribution between NT and EHT-N, while no significant difference was observed between NT and EHT-R or between EHT-N and EHT-R. Furthermore, no significant difference in blood pressure was observed between EHT-R and EHT-N.

As depicted in Table 2, EHT-R showed significantly higher fasting BS (FBS) and summation of BS levels during the OGTT ( $\Sigma$ BS ) than did EHTN. Moreover, EHT-R showed significantly higher IRI levels at 120 min (IRI-120), summation of IRI levels ( $\Sigma$ IRI), and fasting IRI than did NT or EHT-N, while there were no significant differences in IRI levels at 30,60 , or $90 \mathrm{~min}$ (IRI30, IRI60, IRI90) among the three groups during the OGTT. There was no difference in BS levels or IRI levels between NT and EHT-N. On the basis of the results of OGTT, no group satisfied the criterion for diabetes. 
Table 1. Demographic Characteristic of the Groups.

\begin{tabular}{lccc}
\hline & $\begin{array}{c}\text { Normotensive } \\
\text { subjects (NT) } \\
(n=28)\end{array}$ & $\begin{array}{c}\text { Insulin non-resistant } \\
\text { essential hypertensives (EHT-N) } \\
(n=28)\end{array}$ & $\begin{array}{c}\text { Insulin resistant } \\
\text { essential hypertensives (EHT-R) } \\
(n=14)\end{array}$ \\
\hline Male/Female & $20 / 8$ & $11 / 17 *$ & $9 / 5$ \\
Age $(\mathrm{yr}$ old) & $47.0 \pm 2.3$ & $51.0 \pm 2.6$ & $53.1 \pm 3.4$ \\
BMI $\left(\mathrm{kg} / \mathrm{m}^{2}\right)$ & $23.7 \pm 0.4$ & $23.8 \pm 0.5$ & $24.9 \pm 0.5$ \\
Mean blood pressure (mmHg) & $90.3 \pm 1.8$ & $109.5 \pm 1.9 *$ & $105.9 \pm 4.2 *$ \\
$M$ value $\left(\mathrm{mg} / \mathrm{m}^{2} / \mathrm{min}\right)$ & $181.7 \pm 7.3$ & $186.8 \pm 6.3$ & $108.4 \pm 6.0^{*}, \#$ \\
\hline
\end{tabular}

BMI, body mass index. ${ }^{*}: p<0.05 v s$. normotensive subjects, \#: $p<0.05$ vs. insulin non-resistant essential hypertensives.

Table 2. Metabolic Variables According to Group.

\begin{tabular}{|c|c|c|c|}
\hline & $\begin{array}{l}\text { Normotensive } \\
\text { subjects (NT) } \\
\quad(n=28)\end{array}$ & $\begin{array}{c}\text { Insulin non-resistant } \\
\text { essential hypertensives (EHT-N) } \\
(n=28)\end{array}$ & $\begin{array}{c}\text { Insulin resistant } \\
\text { essential hypertensives (EHT-R) } \\
(n=14)\end{array}$ \\
\hline $\mathrm{FBS}(\mathrm{mg} / \mathrm{dl})$ & $87.3 \pm 1.3$ & $84.4 \pm 1.2$ & $90.3 \pm 2.6^{\#}$ \\
\hline$\sum \mathrm{BS}(\mathrm{mg} / \mathrm{dl})$ & $650.1 \pm 36.3$ & $619.9 \pm 19.5$ & $727.2 \pm 42.8^{*}$ \\
\hline F-IRI (mU/l) & $5.3 \pm 0.4$ & $4.1 \pm 0.3^{*}$ & $8.0 \pm 0.8 *$ \\
\hline$\sum$ IRI $(\mathrm{mU} / \mathrm{l})$ & $241.2 \pm 31.2$ & $242.5 \pm 31.9$ & $412.8 \pm 73.1 *$ \\
\hline IRI-120 (mU/l) & $57.0 \pm 9.6$ & $48.7 \pm 5.7$ & $120.7 \pm 28.4^{*}, \#$ \\
\hline $\mathrm{TC}(\mathrm{mg} / \mathrm{dl})$ & $189.2 \pm 5.9$ & $175.1 \pm 5.7$ & $186.2 \pm 8.3$ \\
\hline HDL-C (mg/dl) & $37.2 \pm 2.0$ & $40.6 \pm 2.1$ & $34.1 \pm 2.6$ \\
\hline $\mathrm{TG}(\mathrm{mg} / \mathrm{dl})$ & $102.9 \pm 10.8$ & $88.1 \pm 10.4$ & $180.3 \pm 29.2 *$ \\
\hline FFA $(\mathrm{mg} / \mathrm{dl})$ & $1067.3 \pm 138.7$ & $954.0 \pm 92.9$ & $1480.4 \pm 256.3 \#$ \\
\hline
\end{tabular}

(mean \pm SEM)

FBS, fasting blood sugar; $\Sigma$ BS, summation of blood sugar during the OGTT; F-IRI, fasting immunoreactive insulin level; $\Sigma$ IRI, summation of immunoreactive insulin level during the OGTT; IRI-120, value of immunoreactive insulin at 120 min during the OGTT; TC, total cholesterol; HDL-C, HDL cholesterol; TG triglyceride; FFA, free fatty acid.

*: $p<0.05 v s$. normotensive subjects, \#: $p<0.05$ vs. insulin non-resistant essential hypertensives.

As for plasma lipid values, EHT-R showed significantly higher FFA than EHT-N and higher TG than NT and EHT-N. There was a trend toward lower HDL-C in EHT-R as compared with EHT-N (Table $2)$. There were no differences in lipid values between NT and EHT-N. As shown in Fig. 1, a significant negative correlation, or a trend toward a negative correlation, between $M$-value and FFA ( $r$ $=-0.50, p<0.01)$ or TG $(r=-0.24, p<0.1)$, and significantly positive correlations between IRI-120 and FFA $(r=0.35, p<0.05)$ or TG $(r=0.29, p<$ $0.05)$ were observed in all groups in this study. There was a positive correlation $(r=0.36, p<0.01)$ between $\Sigma$ BS and FFA, while no other significant relation was found between $\Sigma$ BS and serum lipids.

\section{Discussion}

Disturbances of glucose and lipid metabolism are known to be related to hypertension (1-6). Recent studies suggest that insulin resistance and compensatory hyperinsulinemia are linked to these metabolic disturbances. However, not all cases of essential hypertension are accompanied by insulin resistance. Lind et al. (15) first reported the preva- lence of insulin resistance in essential hypertension, estimated with the use of the glucose clamp method, which is the most specific and accurate method available to measure insulin sensitivity. In their study, they divided EHT into insulin-resistant and non-resistant groups, using the mean $-2 \mathrm{SD}$ of the $M$ value of normotensive control subjects as a cutoff point. About $27 \%$ of the EHT were found to be insulin resistant. Furthermore, the EHT-R also showed lipid disturbances in addition to glucose disturbances. However, in their data, EHT-R had significantly higher BMI than EHT-N. Obesity is known to be an independent factor for decreased insulin sensitivity, and obese subjects tend to have accompanying disturbances of glucose and lipid metabolism $(3,17)$. On the other hand, Pollare et al. (18) have reported that insulin resistance is a characteristic feature of essential hypertension, independent of obesity. Therefore, to clarify the association of insulin resistance itself with glucose and lipid metabolism in EHT, it is necessary to conduct a study without the influence of obesity. We therefore studied subjects whose BMIs were lower than $28 \mathrm{~kg} / \mathrm{m}^{2}$, and compared glucose and lipid metabolic variables between EHT-R and BMI-matched NT 

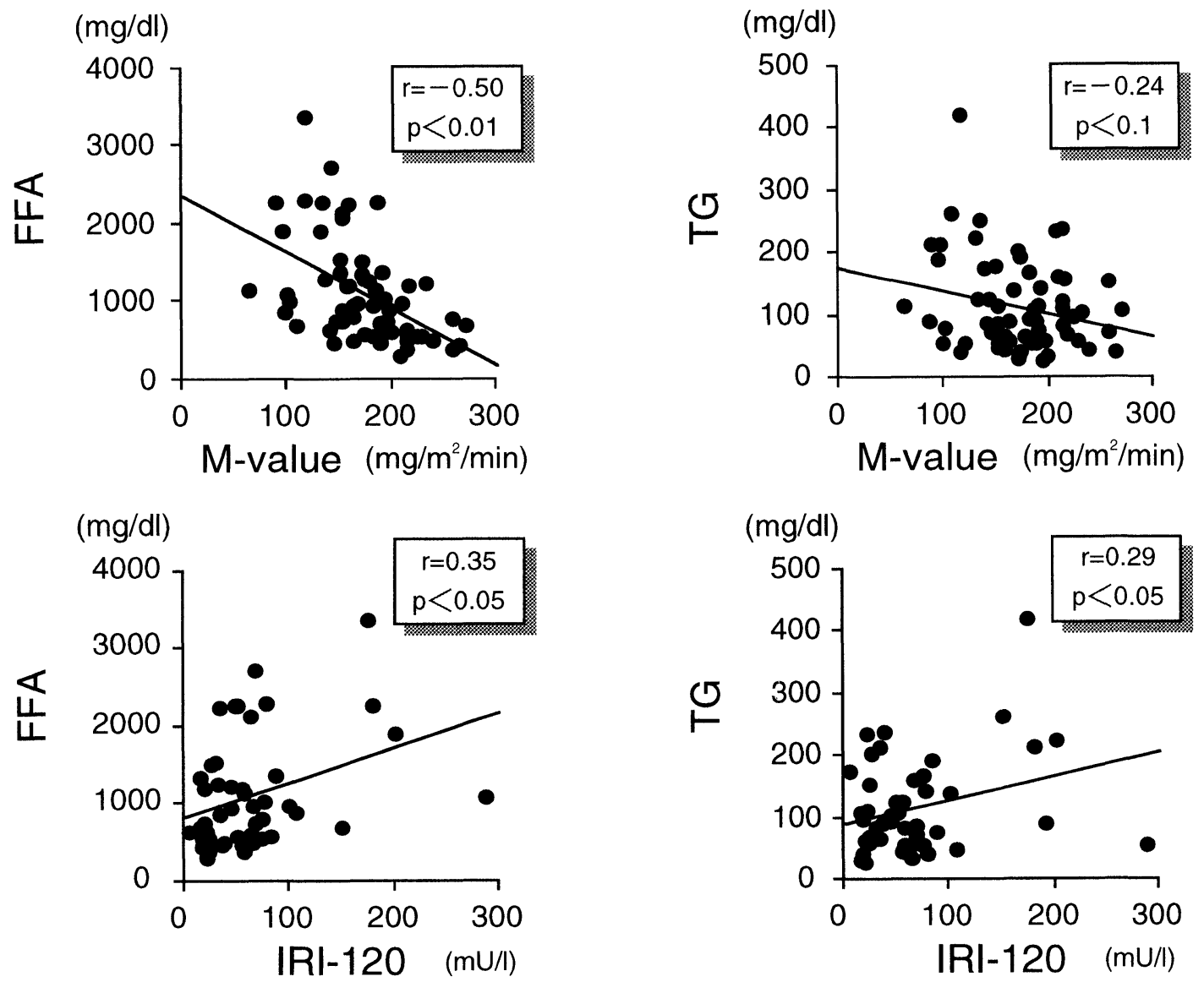

Fig. 1. Correlations between $\mathrm{M}$ value and free fatty acid (FFA) or triglyceride (TG), and correlations between plasma immunoreactive insulin level at $120 \mathrm{~min}$ during the OGTT (IRI-120) and free fatty acid (FFA) or triglyceride (TG).

and EHT-N.

As a cutoff point for insulin resistance, we employed the mean $-1 \mathrm{SD}$ of the $M$ value of NT, whereas Lind et al. (15) used the mean -2SD. In this study, we attempted to exclude the influence of obesity, which is one factor that decreases insulin sensitivity. In non-obese subjects, EHT-R comprised only $19 \%$ of EHT when the mean -2 SD of the $M$ value was used as a cutoff point. Although the mean -1SD of the $M$ value is not an absolute cutoff point, this value may be suitable for distinguishing non-obese EHT-R from EHT-N. In fact, at this cutoff point, $33.3 \%$ of EHT were found to be insulin resistant, although the BMI of these subjects was not higher than that of the other subjects. Furthermore, there was no correlation between $M$ value and BMI in our subjects. These data suggest that, even among non-obese EHT, there were still two subgroups: EHT-R and EHT-N.

Insulin resistance is the reduced ability of insulin to promote glucose uptake in peripheral tissues, and the plasma insulin level increases to compensate for this reduced action of insulin. In this study, EHT-R showed significantly higher levels of F-IRI, $\Sigma$ IRI, IRI-120, FBS, and $\Sigma$ BS during the OGTT as compared with EHT-N and NT. These data indicate that the hyperinsulinemia in EHT-R may be insuffi- cient to compensate for the effect of insulin resistance on glucose metabolism, especially in the postprandial phase.

In our data, there were no significant differences in IRI30, IRI60, or IRI90 among the three groups, while significant differences were observed in IRI120 between EHT-R and NT as well as between EHT-R and EHT-N. We speculate that it may be difficult to detect differences in IRI in the early phase of OGTT, because the secretion of insulin in response to elevated BS may not be decreased even in EHT-R. In other words, IRI levels may depend mainly on the capacity to secrete insulin rather than on insulin resistance in early phase of OGTT. Our data suggest that IRI120 might be best parameter of hyperinsulinemia associated with insulin resistance.

Lind et al. also reported that EHT-R showed evidence of disturbed lipid metabolism as compared with EHT-N; however, they did not study any differences in lipid metabolism in EHT-N as compared with normotensive controls. In our study, EHT-R showed disturbed lipid metabolism as compared with EHT-N and NT. However, there was no significant difference in serum lipid profile between EHT-N and NT. In addition, there was no correlation between $M$ value and BMI because of the narrow range of BMI in our subjects; however, levels 
of TG and FFA correlated negatively with $M$ value and positively with IRI-120 (Fig. 1). In general, insulin resistance, hyperinsulinemia, and hyperglycemia are thought to disturb lipid metabolism. Our data suggest that either insulin resistance or hyperinsulinemia, rather than hyperglycemia, contribute to lipid disturbances; i.e., higher TG and FFA, in EHT without diabetes.

As for the mechanism of lipid disturbances in insulin resistance and compensatory hyperinsulinemia, it has been suggested that (i) hyperinsulinemia enhances hepatic VLDL synthesis and contributes to elevated plasma TG levels (19); (ii) resistance to the action of insulin on lipoprotein lipase also contributes to the increase in the plasma TG level and the decrease in the plasma HDL-C level through the reduced degradation of VLDL (20); (iii) because insulin resistance in peripheral tissue decreases glucose uptake, the plasma FFA level is elevated owing to increased lipolysis in adipose tissues; (iv) the elevated sympathetic nervous activity produced by hyperinsulinemia (11-14) may result in lipolysis and an increase in the plasma FFA level.

We speculate that another cause of lipid disturbances in EHT-R is the difference in fat distribution. Recently, Fujioka et al. (21) reported that intraabdominal visceral fat, which is mainly composed of omental and mesenteric fat, might play a more important pathogenetic role, or might better reflect, underlying metabolic disorders than subcutaneous fat in the development of glucose and lipid disturbances. Therefore, it is possible that visceral fat accumulation may contribute to insulin resistance and lipid disturbances in EHT-R. There were no significant differences in BMI among the three groups in our study; however, we did not investigate the fat distribution. Further investigations, including estimations of fat distribution, are needed to elucidate the precise mechanisms of lipid metabolic disturbances in EHT-R.

No difference in blood pressure between EHT-R and EHT-N was found in this study. However, several reports have already shown that insulin sensitivity is inversely related to blood pressure $(15$, 22). We speculate that there may be subgroups of EHT. In one of these subgroups, an increase in blood pressure due to insulin resistance and hyperinsulinemia would play a part in the pathogenesis of hypertension; in another subgroup it would not. Such a subgroup with insulin resistance and hyperinsulinemia may show a high degree of clustering of other metabolic disturbances.

In summary, (i) $33.3 \%$ of a sample of EHT were found to be insulin resistant, when insulin resistance was defined as an $M$ value $<145.0 \mathrm{mg} / \mathrm{m}^{2} / \mathrm{min}$ (mean -1SD of the $M$ value of NT); (ii) these EHT-R had higher levels of BS, IRI, FFA, and TG than did NT and EHT-N; (iii) EHT-N showed no difference in the levels of BS, IRI, or lipids as compared with NT; and (iv) the levels of FFA and TG correlated negatively with insulin sensitivity and positively with the IRI level during the OGTT.

These findings suggest that essential hypertension often accompanies insulin resistance, compensatory hyperinsulinemia, and resultant disturbances of lipid metabolism, increasing the risks of arteriosclerotic disease in EHT.

\section{References}

1. Jarrett RJ, Keen H, McCartney M, et al: Glucose tolerance and blood pressure in two population samples: their relation to diabetes mellitus and hypertension. Int J Epidemiol 1978; 7: 15-24.

2. Voors AW, Radhakrishnamurthy B, Srinivasan SR, Webber LS, Berenson GS: Plasma glucose level related to blood pressure in 272 children, ages 7-15 years, sampled from a total biracial population. $A m J$ Epidemiol 1981; 113: 347-356.

3. Modan M, Halkin H, Almog S, et al: Hyperinsulinemia: a link between hypertension obesity and glucose intolerance. J Clin Invest 1985; 75: 809-817.

4. Reaven GM: Banting Lecture 1988: role of insulin resistance in human disease. Diabetes 1988; 37: 1595-1607.

5. Kaplan NM: The deadly quartet: upper-body obesity, glucose intolerance, hypertriglyceridemia and hypertension. Arch Intern Med 1989; 149: 1514-1520.

6. DeFronzo RA, Ferrannini E: Insulin resistance: a multifaced syndrome responsible for NIDDM, obesity, hypertension, dyslipidemia, and atherosclerotic cardiovascular disease. Diabetes Care 1991; 14: 173-192.

7. DeFronzo RA, Cooke CR, Andres R, Faloona GR, Davis PJ: The effect of insulin on renal handling of sodium, potassium, calcium, and phosphate in man. $J$ Clin Invest 1975; 55: 845-855.

8. Skött P, Hother-Nielsen O, Bruun NE, et al: Effects of insulin on kidney function and sodium excretion in healthy subjects. Diabetologia 1989; 32: 694-699.

9. Rocchini AP, Katch V, Kveselis D, et al: Insulin and renal sodium retention in obese adolescents. Hypertension 1989; 14: 367-374.

10. Shimamoto $\mathrm{K}$, Nakagawa $\mathrm{M}$, Higashiura $\mathrm{K}$, et al: Insulin sensitivity and renal sodium-water metabolism in obese normotensive subjects. Ann NY Acad Sci 1993; 676: 345-347.

11. Rowe JW, Young JB, Minaker KL, Stevens AL, Pallotta J, Landsberg L: Effect of insulin and glucose infusions on sympathetic nervous system activity in normal man. Diabetes 1981; 30: 219-225.

12. Anderson EA, Hoffman RP, Balon TW, Sinkey CA, Mark AL: Hyperinsulinemia produces both sympathetic neural activation and vasodilation in normal humans. J Clin Invest 1991; 87: 2246-2252.

13. Berne C, Fagius J, Pollare T, Hjemdahl P: The sympathetic response to euglycaemic hyperinsulinaemia. Diabetologia 1992; 35: 873-879.

14. Landsberg L, Young JB: Insulin-mediated glucose metabolism in relationship between dietary intake and sympathetic nervous system activity. Int $J$ Obes 1985; 9 (Suppl 2): 63-68

15. Lind L, Berne C, Lithell $\mathrm{H}$ : Prevalence of insulin resistance in essential hypertension. J Hypertens 1995; 13: $1457-1462$.

16. DeFronzo RA, Tobin JD, Andres R: Glucose clamp technique: a method for quantifying insulin secretion and resistance. Am J Physiol 1979; 237: E214-E223.

17. Landin K, Krotkiewski M, Smith U: Importance of obesity for the metabolic abnormalities associated with an abdominal fat distribution. Metabolism 1989; 38: $572-576$.

18. Pollare $\mathrm{T}$, Lithell $\mathrm{H}$, Berne $\mathrm{C}$ : Insulin resistance is a characteristic feature of primary hypertension inde- 
pendent of obesity. Metabolism 1990; 39: 167-174

19. Olefsky JM, Farquhar JW, Reaven GM: Reappraisal of the role of insulin in hypertriglyceridemia. Am J Med 1974; 57: 551-560.

20. Pollare T, Vessby B, Lithell H: Lipoprotein lipase activity in skeletal muscle is related to insulin sensitivity. Arterioscler Thromb 1991; 11: 1192-1203.
21. Fujioka S, Matsuzawa Y, Tokunaga K, Tarui S: Contribution of intra-abdominal fat accumulation to the impairment of glucose and lipid metabolism in human obesity. Metabolism 1987; 36: 54-59.

22. Saad MF, Liliioja S, Nyomba BL, et al: Racial difference in the relation between blood pressure and insulin resistance. $N$ Engl J Med 1991; 324: 733-739. 